\title{
Can lowering blood pressure prevent vascular complications in patients with type 2 diabetes?
}

\begin{abstract}
Original article Patel A et al. for the ADVANCE Collaborative Group (2007) Effects of a fixed combination of perindopril and indapamide on macrovascular and microvascular outcomes in patients with type 2 diabetes mellitus (the ADVANCE trial): a randomised controlled trial. Lancet 370: 829-840
\end{abstract}

\section{SYNOPSIS}

KEYWORDS angiotensin-converting-enzyme inhibitor, hypertension, indapamide, perindopril, type 2 diabetes

\section{BACKGROUND}

Research suggests that lowering the blood pressure (BP) of patients with type 2 diabetes could reduce the risk of macrovascular and microvascular diabetic complications. Whether this strategy is effective across a range of BP values is not known.

\section{OBJECTIVE}

To establish the efficacy of a fixed combination of indapamide and perindopril at reducing vascular disease in patients with type 2 diabetes.

\section{DESIGN}

The Action in Diabetes and Vascular disease: preterAx and diamicroN MR Controlled Evaluation (ADVANCE) was a multicenter, randomized, double-blind trial. All patients were aged 55 years or older, had been diagnosed with type 2 diabetes after the age of 30 years, and had either a history of major cardiovascular disease (CVD) or an established risk factor for CVD. There were no inclusion or exclusion criteria relating to $\mathrm{BP}$.

\section{INTERVENTION}

Randomization took place after a 6-week run-in period, in which patients received daily a single tablet containing $0.625 \mathrm{mg}$ indapamide and $2.0 \mathrm{mg}$ perindopril. Patients who adhered to and tolerated treatment during run-in were randomly assigned to receive the combined indapamide and perindopril tablet or a matching placebo. Doses were doubled 3 months after randomization. Follow-up visits were scheduled for 3, 4, and 6 months after randomization and at 6-monthly intervals thereafter.

\section{OUTCOME MEASURES}

The primary end points were composites of macrovascular (cardiovascular death, nonfatal stroke, and nonfatal myocardial infarction) or microvascular (diabetes-related blindness, macular edema, proliferative retinopathy, macroalbuminuria, doubling of serum creatinine to at least $200 \mu \mathrm{mol} / \mathrm{l}$, death from renal disease, or the need for retinal photocoagulation or renal replacement therapy) events.

\section{RESULTS}

From 12,877 patients who entered the run-in phase, 11,140 underwent randomization (indapamide plus perindopril $=5,569$; placebo $=5,571)$. At randomization, $32 \%$ of patients had a history of macrovascular disease and $10 \%$ had previous microvascular disease. The mean BP of randomized patients was $145 / 81 \mathrm{mmHg}$. After a mean follow-up of 4.3 years (range $<1$ month to 5.6 years), major macrovascular or microvascular events had occurred in $15.5 \%$ of patients in the indapamide plus perindopril group compared with $16.8 \%$ of those in the placebo group (relative risk reduction $[\mathrm{RRR}] 9 \% ; P=0.041)$. Furthermore, the combined study drugs were associated with lower all-cause mortality than was placebo (7.3\% vs $8.5 \%$, RRR $14 \% ; P=0.025)$, which was primarily attributable to a lower rate of cardiovascular deaths in the combined treatment group (3.8\% vs $4.6 \%$, RRR $18 \% ; P=0.027$ ). Treatment with indapamide and perindopril was also associated with significant reductions in the incidences of renal events $(P<0.0001)$ and microalbuminuria $(P<0.0001)$, and a borderline significant reduction in the incidence of nephropathy $(P=0.055)$.

\section{CONCLUSION}

Combination indapamide and perindopril therapy reduces the risk of macrovascular and microvascular events and death among patients with type 2 diabetes. 


\section{COMMENTARY}

\section{Jan A Staessen}

Modern guidelines for the treatment of hypertension emphasize the global cardiovascular risk of the individual and recommend risk stratification based on BP, target-organ damage, and associated conditions, such as diabetes mellitus. ${ }^{1}$ Hypertension, hypercholesterolemia, and cigarette smoking account for approximately $85 \%$ of the modifiable cardiovascular risk worldwide. ${ }^{2}$ In patients at high-risk of CVD, substantially reducing normal or slightly-elevated cholesterol levels enhances the risk reduction afforded by antihypertensive treatment. ${ }^{2}$ The ADVANCE trial answers the analogous question of whether reducing a normal or only moderately elevated BP might also improve outcomes in high-risk patients. In this study, treatment with indapamide plus perindopril led to an $18 \%$ decrease in cardiovascular mortality and a $9 \%$ reduction in the incidence of microvascular and macrovascular complications when compared with placebo, in unselected patients with diabetes. These findings support the concepts of global cardiovascular risk and multiple risk-factor intervention. ${ }^{1}$

In keeping with the results from large-scale, prospective, observational studies, ${ }^{3}$ a metaregression analysis of randomized clinical trials has demonstrated that small reductions in systolic BP (1-5 mmHg) can explain the differences in cardiovascular outcomes among highrisk patients. ${ }^{4} \mathrm{~A}$ recent meta-analysis has even questioned the effects of angiotensin-convertingenzyme inhibitors and angiotensin-receptor blockers on renal outcomes, over and beyond those attributable to BP reduction per se..$^{5}$ All currently available clinical evidence, therefore, underscores the importance of rigorous $\mathrm{BP}$ control in high-risk patients, regardless of the drug class prescribed. ${ }^{4,5}$ Current guidelines propose a BP threshold of below $130 / 80 \mathrm{mmHg}$ in high-risk patients, such as those with diabetes mellitus. ${ }^{1}$ Results from the ADVANCE trial support this recommendation.

At first sight, the importance of BP reduction in the prevention of the cardiovascular complications of hypertension indicates a generic approach to treatment, supporting the role of low-dose combinations of antihypertensive agents. Additional arguments in favor of such depersonalized treatment are that most patients require more than a single drug for adequate $\mathrm{BP}$ control, and that low-dose combinations expose patients to fewer adverse effects and might, therefore, increase adherence to therapy. Stroke is the complication most directly associated with elevated $\mathrm{BP} .^{3}$ In the ADVANCE trial, patients receiving active treatment had an average $5.6 \mathrm{mmHg}$ reduction in systolic BP compared with those on placebo. For the $5.6 \mathrm{mmHg}$ reduction in systolic $\mathrm{BP}$ observed in the ADVANCE trial, the observed and predicted ${ }^{4}$ risk ratios (active/placebo) for major cerebrovascualar events were 0.98 (95\% CI 0.82-1.19) and 0.68 (95\% CI $0.62-0.75),{ }^{4}$ respectively $(P$ for difference $=0.0007)$. After PROGRESS and EUROPA, ADVANCE is the third perindoprilbased trial in which stroke prevention was poor relative to BP reduction. Expert committees now have to consider whether the convenience and low cost of the generic approach, as proposed in the ADVANCE trial, outweigh the advantages of the laborious, but intellectually more attractive, individualized strategy of starting with one drug and optimizing therapy by substitution or addition of other compounds. Most of the systolic BP gradient seen in the ADVANCE trial was caused by an increase in $\mathrm{BP}$ among patients receiving placebo (from 137 to $140 \mathrm{mmHg}$ ) rather than a decrease in $\mathrm{BP}$ among those in the active-treatment group (from 137 to $136 \mathrm{mmHg}$ ). What clinicians should, therefore, remember from the ADVANCE trial is not to stop antihypertensive therapy in diabetic patients, but to prescribe such drugs on an individualized basis until target $\mathrm{BP}$ is achieved.

\section{References}

1 Mancia G et al. (2007) 2007 Guidelines for the management of arterial hypertension: the Task Force for the Management of Arterial Hypertension of the European Society of Hypertension (ESH) and of the European Society of Cardiology (ESC). Eur Heart J 28: 1462-1536

2 Staessen JA et al. (2007) 'Beyond blood pressure' means multiple risk factor intervention, not pleiotropic antihypertensive drugs. Curr Opin Cardiol 22: 335-343

3 Lewington Set al. for the Prospective Studies Collaboration (2002) Age-specific relevance of usual blood pressure to vascular mortality: a metaanalysis of individual data for one million adults in 61 prospective studies. Lancet 360: 1903-1913

4 Staessen JA et al. (2001) Cardiovascular protection and blood pressure reduction: a meta-analysis. Lancet 358: $1305-1315$

5 Casas JP et al. (2005) Effects of inhibitors of the renin-angiotensin system and other antihypertensive drugs on renal outcomes: systematic review and meta-analysis. Lancet 366: 2026-2033

\section{JA Staessen heads the Study Coordinating}

Centre, Hypertension Unit in the Department of Cardiovascular Diseases, University of Leuven, Leuven, Belgium.

Acknowledgments

The synopsis was written by Alexandra King, Associate Editor,

Nature Clinical Practice.

\section{Competing interests The author has declared associations with the following companies: Daiichi Sankyo, Mitsubishi Tanabe Pharma, Pfizer, and Sigma-Tau. See the article online for full details of the relationships.}

\section{Correspondence \\ Study Coordinating Centre \\ Division of Hypertension and \\ Cardiovascular Rehabilitation \\ Department of \\ Cardiovascular Diseases \\ University of Leuven- \\ Gasthuisberg Campus \\ Box 702 \\ Herestraat 49 \\ B-3000 Leuven \\ Belgium \\ jan.staessen@ \\ med.kuleuven.be}

Received 3 November 2007 Accepted 27 November 2007 Published online 29 January 2008

www.nature.com/clinicalpractice doi:10.1038/ncpcardio1124

PRACTICE POINT

For the prevention of cardiovascular complications in diabetic and nondiabetic patients alike, lowering blood pressure is what counts, not the way in which it is lowered 